\title{
Coevolutionary Genetic Algorithm Based on the Augmented Lagrangian Function for Solving the Economic Dispatch Problem
}

\author{
L. Nepomuceno, Member, IEEE, E. C. Baptista, A. R. Balbo, E. M. Soler
}

\begin{abstract}
This paper proposes a coevolutionary augmented Lagrangian method (AGCE) for solving the classic economic dispatch problem. This problem becomes non-convex and nondifferentiable if valve-point loadings effects are considered in the cost curves of thermal units. In such cases, the evolutionary approaches have proven to be efficient for solving the primal economic dispatch problem; however, the great majority of these methods are not capable of solving the associated dual problem. Furthermore, the solutions obtained by these methods cannot be evaluated concerning their optimality. The AGCE works in the primal-dual subspaces and is able to calculate both primal and dual optimal values. For such a purpose, AGCE processes, in parallel, the evolution of two distinct groups of individuals, associated with primal and dual variables, respectively. The "clouds" of primal and dual points become iteratively denser, and converge to the saddle points associated with the problem, even in the presence of non-differentiability points. Therefore, AGCE makes possible the evaluation of optimality of its solution points. In the results, the AGCE is compared with a traditional interior point method and with a genetic algorithm that works only in the primal subspace.
\end{abstract}

Keywords-Genetic algorithms, evolutionary computation, augmented Lagrangian method, economic dispatch.

\section{INTRODUÇÃO}

$\mathrm{O}$ PROBLEMA de despacho econômico (DE) tem como objetivo o cálculo da potência de saída de unidades de geração em um determinado intervalo de tempo do dia, bem como o preço da energia neste intervalo. O DE, que é básico para o planejamento e operação de sistemas de energia, tem sido resolvido por meio de várias técnicas computacionais, que envolvem desde métodos de otimização convencionais, tais como o método de pontos interiores (MPI) [12] [6], até métodos baseados em técnicas de inteligência computacional, tais como redes neurais [17], algoritmos genéticos [1], dentre outros.

Quando a função objetivo do DE que envolve os custos de geração é formulada de modo a representar os efeitos de pontos de válvula, ela assume características de nãoconvexidade e não-diferenciabilidade em certos pontos, tornando a solução do DE mais complexa e dificultando a aplicação de técnicas convencionais. Nesses casos, o problema

L. Nepomuceno, Unesp - Univ Estadual Paulista, Brasil, Departamento de Engenharia Eléctrica, Brasil, leo@feb.unesp.br.

E. C. Baptista, Unesp - Univ Estadual Paulista, Brasil, Departamento de Matemática, Brasil, baptista@fc.unesp.br.

A. R. Balbo, Unesp - Univ Estadual Paulista, Brasil, Departamento of Matemática, Brasil, arbalbo@fc.unesp.br.

E. M. Soler, Unesp - Univ Estadual Paulista, Brasil, Departamento of Matemática, Brasil, edilaine@fc.unesp.br. tem sido resolvido por heurísticas[19], tais como computação evolutiva [22], algoritmos genéticos [2][21], enxame de partículas [4][7], sistemas de formiga [5], sistemas imunes artificiais [8], algoritmos com inspiração quântica [10][11], busca tabu [16], simulated annealing [20], evolução diferencial [14], etc. Todas estas técnicas trabalham no espaço das variáveis primais do problema, e não conseguem resolver o problema dual associado. Outra deficiência destas metodologias é a impossibilidade de verificação quanto à otimalidade da solução. Em geral, estes métodos evoluem uma população de indivíduos, por uma quantidade estipulada de gerações, e o critério de parada não se baseia na otimalidade.

Neste trabalho, busca-se resolver os problemas primal e dual associados ao DE. As vantagens de se calcular a solução primal-dual para o problema são as seguintes: i) as variáveis duais fornecem importantes interpretações econômicas associadas ao problema (e.g., a variável dual associada à restrição de atendimento de demanda representa o preço spot da energia); ii) as variáveis duais permitem a análise de sensibilidade na vizinhança da solução ótima, evitando a resolução do problema para pequenas perturbações; iii) com a utilização da variáveis duais, pode-se converter o problema primal restrito em um problema dual irrestrito, através da utilização da função Lagrangiana aumentada. Assim, a abordagem primal dual permite tratar as restrições de igualdade e desigualdade por meio de estratégias evolutivas; iv) na abordagem primal dual, é possível calcular o gap de dualidade, que é uma medida da otimalidade da solução e este pode ser utilizado como critério de parada do método.

Neste trabalho, propõe-se a solução do problema de DE por meio de um Algoritmo Genético Co-Evolutivo (AGCE) baseado na abordagem da função Lagrangiana aumentada, descrita em [18]. O AGCE utiliza a função Lagrangiana aumentada como função de avaliação e processa a evolução de dois grupos de indivíduos em paralelo, os quais estão associados às variáveis primais e duais, respectivamente. Como critério de parada este trabalho propõe a avaliação do gap de dualidade entre as populações primais e duais, o qual envolve a definição do gap de dualidade agrupado, calculado entre as populações primal e dual. As "nuvens" de pontos primais e duais convergem para pontos de sela do problema, os quais estão associados à solução do DE, mesmo quando da existência de pontos de não diferenciabilidade, conforme mostrado nos resultados. Além disso, o gap de dualidade agrupado converge para o gap de dualidade convencional na solução ótima.

Os resultados apresentados comparam a solução do AGCE com método de pontos interiores, estado da arte para a solução do problema, e com um método de AG convencional, o qual 
trabalha apenas no espaço primal de solução do problema. Mostra-se que é possível calcular os despachos e as variáveis duais do $\mathrm{DE}$ mesmo quando da ocorrência de pontos de não diferenciabilidade causados pela inserção de pontos de válvula na função de custo.

\section{MÉtodos Baseados nA LAgRAngianA AumentadA}

O método da Lagrangiana aumentada foi introduzido de forma independente por Hestenes em [9] e Powell em [13] para problemas de otimização envolvendo restrições de igualdade e estendido por Rockafellar [15] para problemas envolvendo restrições de desigualdade. Por facilidade, as principais ideias relacionadas aos métodos baseados na função Lagrangiana aumentada para a solução de problemas de otimização não linear são resumidos nesta seção. Para isso, considere o problema de otimização restrito primal descrito de (1) a (4), sobre $x \in \mathfrak{R}^{n}$ :

$$
\operatorname{Min}_{x} f(x)
$$

sujeito a:

$$
\begin{aligned}
& g_{i}(x) \leq 0 \quad \forall i=1, \cdots, m \\
& h_{j}(x)=0 \quad \forall j=1, \cdots, l \\
& x_{l}^{\min } \leq x_{l} \leq x_{l}^{\max } \quad \forall l=1, \cdots, n
\end{aligned}
$$

Seja $S \subset \mathfrak{R}^{n}$ o subespaço de busca especificado por (4). Para o problema primal, tem-se o problema dual associado, conforme descrito em (5) e (6):

$$
\underset{\mu, \lambda}{\operatorname{Max}} \theta(\mu, \lambda)
$$

sujeito a:

$$
\mu_{i} \geq 0 \quad \forall i=1, \cdots, m,
$$

em que:

$$
\theta(\mu, \lambda)=\min _{x}\left\{f(x)+\mu^{T} g(x)+\lambda^{T} h(x)\right\}, x \in S .
$$

Os vetores $\mu \in \mathfrak{R}^{m}$ e $\lambda \in \mathfrak{R}^{l}$ são os multiplicadores de Lagrange associados às restrições (2) e (3), respectivamente. Se o problema primal é convexo sobre $S \subset \mathfrak{R}^{n}$, o teorema da dualidade forte [3] garante que:

$$
\begin{aligned}
& \min _{x}(f(x): g(x) \leq, h(x)=0, x \in S) \\
& =\max _{\mu, \lambda}(\theta(\mu, \lambda): \mu \geq 0) .
\end{aligned}
$$

Nesse caso, a solução $x^{*}$ do problema primal juntamente com a solução $\left(\mu^{*}, \lambda^{*}\right)$ do problema dual satisfazem as condições de Karush-Kuhn-Tucker (KKT), e correspondem ao ponto de sela da função Lagrangiana descrita em (9):

$$
L(x, \mu, \lambda)=f(x)+\mu^{T} g(x)+\lambda^{T} h(x) .
$$

Assim, pode-se escrever (10):

$$
L\left(x^{*}, \mu, \lambda\right) \leq L\left(x^{*}, \mu^{*}, \lambda^{*}\right) \leq L\left(x, \mu^{*}, \lambda^{*}\right)
$$

para todo $x \in S$ e $\mu \geq 0$. Nota-se que o lado direito da desigualdade implica que $x^{*}$ é o mínimo irrestrito de $L\left(x, \mu^{*}, \lambda^{*}\right)$, ou seja, se $\left(\mu^{*}, \lambda^{*}\right)$ é conhecido, pode-se buscar $x^{*}$ em $S$ sem considerar as restrições (2) e (3). Para esse caso, pode-se também provar [3] que a solução da minimização irrestrita de (9) sobre $S \subset \mathfrak{R}^{n}$ é equivalente à solução do problema primal.

Por outro lado, quando o problema não é convexo sobre $S \subset \mathfrak{R}^{n}$, pode existir um gap de dualidade entre os problemas primal e dual. Para os casos em que o gap de dualidade existe, o teorema da dualidade fraca [3] garante que (11) ocorre, porém não existe neste caso um ponto de sela que satisfaça (10):

$$
\begin{aligned}
& \min _{x}(f(x): g(x) \leq, h(x)=0, x \in S) \\
& >\max _{\mu, \lambda}(\theta(\mu, \lambda): \mu \geq 0) .
\end{aligned}
$$

Além disso, uma busca irrestrita de $x^{*}$ em $S$ não é possível, mesmo que $\left(\mu^{*}, \lambda^{*}\right)$ seja conhecido. Os métodos baseados na função Lagrangiana aumentada evitam esse problema tornando $f$ convexa com a inserção de termos de penalidade quadrática associados às restrições (2) e (3). A função Lagrangiana aumentada associada ao problema dado de (1) a (4) é geralmente definida conforme (12) [3]:

$$
\begin{gathered}
L_{A}(x, \mu, \lambda, \rho)= \\
f(x)+\sum_{k=1}^{m} p_{k}\left(x, \mu_{k}, \rho\right)+\lambda^{T} h(x)+\rho \sum_{k=1}^{l} h_{k}(x)^{2},
\end{gathered}
$$

em que $\lambda$ e $\rho$ são, respectivamente, os multiplicadores de Lagrange e a penalidade, associados às restrições de igualdade; o termo de penalidade $p_{k}\left(x, \mu_{k}, \rho\right)$, relacionado às restrições de desigualdade, é dado conforme (13):

$$
p_{k}\left(x, \mu_{k}, \rho\right)=\left\{\begin{array}{ll}
\mu_{i} g_{i}(x)+\rho g_{i}^{2}(x) \text { se } g_{i}(x) \geq-\frac{\mu_{i}}{2 \rho} \\
-\frac{\mu_{i}^{2}}{4 \rho} \quad \text { se } g_{i}(x)<-\frac{\mu_{i}}{2 \rho}
\end{array},\right.
$$

em que $\mu_{i}$ e $\rho$ são, respectivamente, o multiplicador de Lagrange e o termo de penalidade. Mostra-se também [3] que a solução do problema de minimização irrestrita de (12) corresponde à solução do problema primal $\left(x^{*}, \mu^{*}, \lambda^{*}\right)$. Sabese ainda que se a solução de KKT é um mínimo local forte, então existe um $\bar{\rho}$ tal que $x^{*}$ é um mínimo local forte de $L_{A}\left(x, \mu^{*}, \lambda^{*}, \rho\right)$ para todo $\rho \geq \bar{\rho}$; a Hessiana de $L_{A}$ com respeito a $x$ próximo à solução $\left(x^{*}, \mu^{*}, \lambda^{*}\right)$ pode ser feita definida positiva. Portanto por meio da utilização da função Lagrangiana aumentada, pode-se mais uma vez calcular $x^{*}$ 
por meio de uma busca irrestrita se $\left(\mu^{*}, \lambda^{*}\right)$ for conhecido e a busca se inicie em um ponto próximo a $x^{*}$.

Os métodos baseados na função Lagrangiana aumentada possuem duas vantagens em relação a outros métodos de otimização não-linear. Primeiro, a solução exata pode ser encontrada, o que não é verdade para os métodos de funções de penalidade simples. Segundo, a direção de busca não é influenciada pelas restrições (2) e (3), já que $x^{*}$ é o mínimo irrestrito de $L_{A}\left(x, \mu^{*}, \lambda^{*}, \rho\right)$ na vizinhança de $x^{*}$. A questão mais importante relacionada aos métodos de otimização determinísticos baseados na Lagrangiana aumentada é como atualizar o multiplicador de Lagrange de modo que o método convirja para $\left(\mu^{*}, \lambda^{*}\right)$. Ao contrário dessas abordagens, a classe de métodos co-evolutivos proposta em [18], utiliza uma abordagem que evolui tanto $(\mu, \lambda)$ quanto $x$ para atingir o ponto de sela $\left(x^{*}, \mu^{*}, \lambda^{*}\right)$. O AGCE proposto neste trabalho, o qual é descrito na seção a seguir, se baseia na plataforma coevolutiva descrita em [18].

\section{Algoritmo Genético Co-Evolutivo}

$\mathrm{O}$ algoritmo genético co-evolutivo (AGCE) é baseado na evolução de dois grupos de indivíduos com objetivos distintos: o primeiro grupo (grupo primal) é composto de soluções candidatas $x_{i}$ ao problema primal; o segundo (grupo dual) é composto pelas soluções candidatas $\left(\mu_{j}, \lambda_{j}\right)$ ao problema dual. Conforme comentado na seção anterior, a utilização da função Lagrangiana aumentada nos permite encontrar a solução primal $x^{*}$ através de uma busca irrestrita em $S \subset \mathfrak{R}^{n}$, desde que a solução ótima dual $\left(\mu^{*}, \lambda^{*}\right)$ seja conhecida; por outro lado, a solução ótima dual $\left(\mu^{*}, \lambda^{*}\right)$ também pode ser calculada através de uma busca irrestrita para $\mu \geq 0$, desde que $x^{*}$ seja conhecido. Entretanto, nem $\left(\mu^{*}, \lambda^{*}\right)$ é conhecido no processo evolutivo utilizado para resolver o problema primal, nem $x^{*}$ é conhecido no processo evolutivo utilizado para resolver o problema dual. Para resolver este problema, a plataforma co-evolutiva descrita em [18] propõe uma jogo matricial de dimensão finita o qual consiste em uma aproximação de um jogo de soma-nula. Nesta abordagem, o processo evolutivo utilizado para a solução do problema primal considera todos os candidatos $\left(\mu_{j}, \lambda_{j}\right)$ do grupo dual, estabelecendo um jogo matricial de dimensão finita. $O$ processo evolutivo para a solução do problema dual passa por um processo análogo. $\mathrm{O}$ processo evolutivo torna o jogo matricial cada vez melhor, no sentido de que cada grupo possui uma população mais densa em torno do ponto de sela do problema. A avaliação da adaptabilidade é baseada em uma estratégia de segurança de cada grupo, que corresponde a uma estratégia básica de jogos matriciais de soma nula. As principais ideias envolvendo jogos matriciais e co-evolução são resumidas a seguir.

Seja um jogo estático de soma nula $G$ para o qual a função de payoff $F=F(u, v)$ deve ser minimizada em $u \in U$ e maximizada em $v \in V$. Assume-se que existe um par $\left(u^{*} \in U, v^{*} \in V\right)$ tal que:

$$
\min _{u} F(u, v)=\max _{v} F(u, v)=F\left(u^{*}, v^{*}\right)=F^{*} .
$$

Assim, o ponto $\left(u^{*}, v^{*}\right)$, que é chamado de solução de ponto de sela, satisfaz (15):

$$
F\left(u^{*}, v\right) \leq F\left(u^{*}, v^{*}\right) \leq F\left(u, v^{*}\right), \forall u \in U, \forall v \in V .
$$

Suponha agora que $u$ e $v$ possuam um número finito de opções conforme (16) e (17), respectivamente:

$$
\begin{aligned}
& U=\left\{u_{1}, u_{2}, \cdots, u_{N_{u}}\right\} \\
& V=\left\{v_{1}, v_{2}, \cdots, v_{N_{v}}\right\},
\end{aligned}
$$

onde $N_{u}$ e $N_{v}$ são as opções de $u$ e $v$ respectivamente. Assim, a tripla $(F, U, V)$ define um jogo matricial $G$. O jogo matricial pode ser interpretado no contexto coevolutivo da seguinte forma: $U$ é um conjunto que contém $N_{u}$ elementos $u_{i}$, os quais representam os indivíduos codificados $x_{i}$ do problema primal (candidatos à solução do problema primal); $V$ é um conjunto que contém $N_{v}$ elementos $v_{j}$, que representam os indivíduos codificados $\left(\mu_{j}, \lambda_{j}\right)$ do problema dual (candidatos à solução do problema dual). $\mathrm{O}$ resultado do jogo $\left(u_{i}, v_{j}\right)$ é definido como o valor da função de payoff $F\left(u_{i}, v_{j}\right)$. A função Lagrangiana aumentada (12) é utilizada como função de payoff na abordagem AGCE.

Assim, resolver o jogo $G$ a cada geração corresponde a encontrar o melhor indivíduo de cada grupo através da avaliação das funções de payoff. $F\left(u_{i}, v_{i}\right)$ de cada elementos dos grupos primal e dual. Para o grupo primal, o melhor indivíduo $u_{i}$ é aquele que minimiza a função de payoff (função Lagrangiana aumentada). Entretanto, a função de payoff também é função dos indivíduos duais $v_{i}$. Como a opção do melhor indivíduo $v_{i}$ do grupo dual é desconhecida, a análise do melhor indivíduo primal deve considerar a pior situação relacionada a cada opção dual, ou seja, deve considerar aquele que fornece o maior valor para a função de payoff. (estratégia de segurança no jogo). Portanto, o valor de fitness fit $_{u_{i}}$ para um determinado indivíduo $u_{i}$ (representando $x_{i}$ ) é determinado utilizando-se o máximo valor de payoff obtido nos $N_{v}$ jogos $\left(u_{i}, v_{j}\right) ; j=1, \cdots, N_{v}$. Como o problema primal é de minimização, o valor da função de fitness fit $t_{u_{i}}$, a qual é inversamente proporcional à função de payoff $F\left(u_{i}, v_{j}\right)$, é calculada conforme (18):

$$
\text { fit }_{u_{i}}=\frac{1}{\max _{j} F\left(u_{i}, v_{j}\right)}, \forall i=1, \cdots, N_{u} .
$$


De forma análoga, como a opção do melhor indivíduo $u_{i}$ do grupo primal não é conhecida pelo grupo dual, o melhor indivíduo para o grupo dual é calculado considerando-se o pior caso de todas as $u_{i}, \forall i=1, \cdots, N_{u}$ opções. A pior opção para o problema dual, que envolve a maximização de $F\left(u_{i}, v_{i}\right)$, é aquela com menor valor de função payoff. (estratégia de segurança no jogo). Portanto, o valor de fitness fit $_{v_{j}}$ para um indivíduo dual $v_{j}$ (representando $\left(\mu_{j}, \lambda_{j}\right)$ ) é determinado utilizando-se o mínimo valor de payoff obtido $\operatorname{nos} N_{u}$ jogos $\left(u_{i}, v_{j}\right) ; i=1, \cdots, N_{u}$. Como o problema dual é de maximização, o valor da função de fitness é diretamente proporcional a $F\left(u_{i}, v_{j}\right)$. Assim, o valor de $f_{i t_{j}}$ para um indivíduo $v_{j}$ é calculado conforme mostrado em(19):

$$
\text { fit }_{v_{j}}=\min _{i} F\left(u_{i}, v_{j}\right), \forall j=1, \cdots, N_{v} .
$$

A Fig. 1 mostra o fluxograma do AGCE, que consiste basicamente na evolução em paralelo de dois grupos de populações de indivíduos. As funções de fitness, definidas em (18) e (19) com base na estratégia de segurança, estabelecem uma ligação entre os processos evolutivos para os dois grupos.

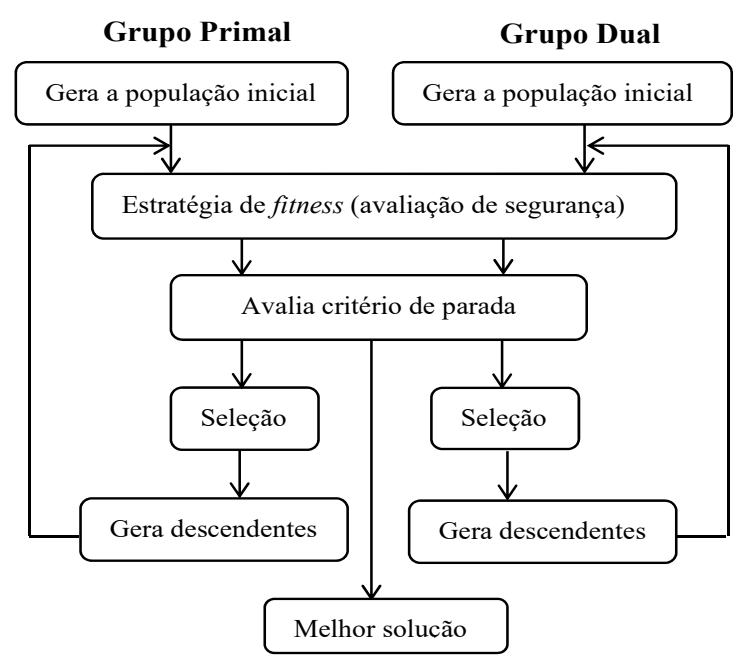

Figura 1. Fluxograma do método co-evolutivo AGCE (Adaptado da Figura descrita em [18]).

Em [18], propõe-se um algoritmo de estratégia evolutiva para resolver os processos de seleção, recombinação e mutação de cada grupo populacional mostrado na Fig. 1. No artigo aqui proposto, utilizamos um AG com codificação em real (AGCE), que será descrito na seção 5. Na seção 4, descrevemos o problema de despacho econômico que será utilizado para a avaliação do AGCE proposto.

\section{Problema de Despacho ECONÔMICO}

O problema de despacho econômico clássico (DE) consiste em calcular uma política de despacho de geração que minimiza os custos de geração e respeita as restrições de atendimento de demanda e limites operacionais das unidades geradoras, conforme descrito de (20) a (22):

$$
\operatorname{Min} C_{T}(p)=\sum_{t=1}^{n t} C_{t}\left(p_{t}\right)
$$

sujeito a:

$$
\begin{aligned}
& \sum_{t=1}^{n t} p_{t}=D \\
& p_{t}^{\min } \leq p_{t} \leq p_{t}^{\max } ; t=1, \cdots, n t,
\end{aligned}
$$

em que:

$t$ : índice para as unidades termelétricas;

$n t$ : número de unidades termelétricas;

$p_{t}$ : $\quad$ potência ativa gerada pela unidade termelétrica $t$;

$p: \quad$ vetor de potência gerada por todas as unidades;

$C_{T}(p):$ custo total de geração;

$C_{t}\left(p_{t}\right)$ : custo de geração da unidade termelétrica $t$;

$D$ : $\quad$ demanda total do sistema;

$p_{t}^{\text {min }}: \quad$ limite de geração mínima para a unidade $t$;

$p_{t}^{\max }: \quad$ limite de geração máxima para a unidade $t$.

Em uma formulação mais geral, o problema de DE pode incluir as perdas na transmissão bem como a representação de reservas girantes. A inclusão destas restrições torna o problema mais representativo, entretanto, para fins de avaliação da metodologia de solução proposta, neste trabalho adota-se o modelo de DE clássico descrito de (20) a (22).

A função de produção das unidades termelétricas envolve os custos de combustíveis necessários à geração da energia. Esta função é descrita em (23), e mostrada na Figura 2.

$$
C_{t}\left(p_{t}\right)=a_{t} p_{t}^{2}+b_{t} p_{t}+c_{t}+\left|e_{t} \operatorname{sen}\left(f_{t}\left(p_{t}^{\min }-p_{t}\right)\right)\right|
$$

em que $a_{t}, b_{t}, c_{t}, e_{t}, f_{t}$ são os coeficientes da função de produção. Quando os chamados efeitos de pontos de carregamento de válvula são considerados na função de produção, temos $e_{t} \neq 0, f_{t} \neq 0$. Neste caso, a função objetivo torna-se não convexa e não diferenciável, com vários máximos e mínimos locais, conforme mostrado na Fig. 2. Em muitas aplicações, os efeitos de pontos de válvula são desprezados, fazendo-se $e_{t}=f_{t}=0$. Neste caso, tem-se a função quadrática, mais simples, também mostrada na Figura 2.

A introdução dos efeitos de pontos de carregamento de válvula na função de custo complica bastante a solução do problema de otimização, já que a maioria das técnicas de solução convencionais utiliza informações do gradiente (ou baseadas no gradiente), o qual, em função da não diferenciabilidade da função, não é diretamente calculável. Em razão disso, procedimentos de otimização baseados em diferentes tipos de algoritmos evolutivos, os quais não necessitam de garantias de convexidade ou diferenciabilidade das funções, têm sido aplicados para resolver o problema de DE não convexo. Entretanto, estes algoritmos não são capazes de calcular as variáveis duais associadas ao problema, limitando sua utilização prática. Na seção a seguir, apresentase a aplicação do AGCE proposto para resolver os problemas primal e dual associados ao DE. 


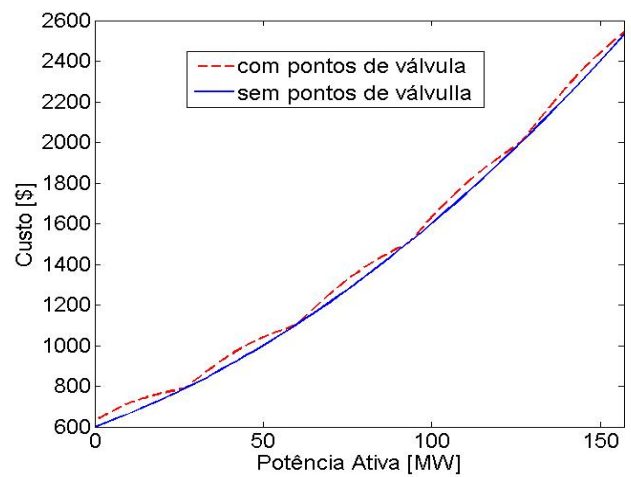

Figura 2. Função de custo de produção para uma unidade termelétrica com e sem a representação de pontos de válvula.

\section{Algoritmos Genéticos Co-Evolutivos PARA A SOlUÇÃO DO PROBlEMA DE DESPACHO ECONÔMICO}

Seja o problema de DE primal, formulado conforme (20) a (22). Definindo $S \subset \Re^{n}$ como o subespaço definido pelas restrições (22) a função Lagrangiana aumentada associada ao problemas é escrita conforme (24):

$$
L_{A}(p, \lambda, \rho)=C_{T}(p)+\lambda\left(\sum_{t=1}^{n t} p_{t}-D\right)+\rho\left(\sum_{t=1}^{n t} p_{t}-D\right)^{2},
$$

em que $\lambda$ é o multiplicador de Lagrange associado a (21) e $\rho$ é o parâmetro de penalidade

O problema dual associado ao problema primal é mostrado em (25), em que $\phi(\lambda)$ é dado em (26):

$$
\begin{gathered}
\operatorname{Max}_{\lambda} \phi(\lambda) \\
\phi(\lambda)= \begin{cases}\operatorname{Min}_{p} & L_{A}(p, \lambda) \\
\text { s.t. }: & \\
& p \in S .\end{cases}
\end{gathered}
$$

No AGCE proposto, os processos evolutivos para a solução dos problemas primal e dual são conduzidos de forma individualizada, conforme descrito na Fig. 1. No problema de DE, um indivíduo $X_{u}$ do grupo primal representa o despacho de geração e é descrito em (27), enquanto que um indivíduo do grupo dual $Y_{v}$ representa o preço da energia e é descrito conforme (28), em que $N_{u}$ e $N_{v}$ correspondem ao número de indivíduos nos grupos primal e dual, respectivamente. Tanto $X_{u}$ quanto $Y_{v}$ são codificados em real:

$$
\begin{gathered}
X_{u}=(p)^{u} ; \quad u=1,2, \cdots, N_{u} \\
Y_{v}=(\lambda)^{v} ; \quad v=1, \cdots, N_{v} .
\end{gathered}
$$

Utilizando-se as estruturas codificadas em real dadas em (27) e (28), o AGCE utiliza a abordagem evolutiva descrita na Fig. 1 para calcular as soluções ótimas dos problemas primal e dual. Detalhes dos operadores genéticos específicos para o problema de DE são discutidos a seguir.

\section{A. Geração da População Inicial}

Dois grupos de indivíduos codificados em real $X_{u}$ e $Y_{v}$, contendo respectivamente $N_{u}$ e $N_{v}$ indivíduos, são randomicamente gerados, de modo que $X_{u} \in S ; u=1, \cdots, N_{u}$ e $Y_{v} ; v=1, \cdots, N_{v}$ possui valores irrestritos.

\section{B. Avaliação do Fitness}

Os valores de fitness são calculados utilizando-se o conceito da estratégia de segurança (escolha do pior caso), em um jogo matricial, conforme descrito na seção 3. Para isso, as equações (18) e (19) são utilizadas, e a função Lagrangiana aumentada (24) é utilizada como função de avaliação. Este procedimento resulta nas funções de fitness descritas em (29) e (30) para indivíduos dos grupos primal e dual, respectivamente:

$$
\begin{gathered}
F u_{u}\left(X_{u}\right)=\frac{1}{\max _{v} L_{A}\left(X_{u}, Y_{v}\right)} ; \forall u=1, \cdots, N_{u} \\
F v_{v}\left(Y_{v}\right)=\min _{u} L_{A}\left(X_{u}, Y_{v}\right) ; \forall v=1, \cdots, N_{v},
\end{gathered}
$$

em que:

$F u_{u}\left(X_{u}\right)$ : fitness para o indivíduo do grupo primal $X_{u}$;

$F v_{v}\left(Y_{v}\right)$ : fitness para o indivíduo do grupo dual $Y_{v}$.

\section{Mecanismo de Seleção}

A partir do mecanismo de seleção, escolhem-se os melhores indivíduos para compor a próxima geração. Qualquer método de seleção pode ser teoricamente utilizado. Neste trabalho, utiliza-se um algoritmo de roleta viciada.

\section{Critério de Parada}

Neste trabalho, utiliza-se o conceito de gap de dualidade como o critério de parada para o AGCE. Como o problema de DE é resolvido por um método co-evolutivo, que trabalha no espaço primal-dual de indivíduos, ao invés de no espaço primal dual de pontos de solução, como em otimização tradicional, é necessário estabelecer um novo conceito para o gap de dualidade, o qual é definido a seguir.

Em otimização não-linear tradicional, o gap de dualidade é medido como a diferença entre os valores da função objetivo para os problemas primal e dual, calculados em seus respectivos pontos ótimos $p^{*}$ e $\lambda^{*}$. Para o problema de DE dado de (20) a (22), o gap de dualidade é dado por (31):

$$
\text { gap }=C_{T}\left(p^{*}\right)-L_{A}\left(p^{*}, \lambda^{*}\right) .
$$

Quando tem-se gap $=0$, os valores das funções objetivo dos problemas primal e dual avaliados em seus respectivos pontos ótimos possuem o mesmo valor.

Suponha que existe um ponto de sela que resolve o problema de DE, de modo que (32) seja válida:

$$
\min _{p} L_{A}\left(p, \lambda^{*}\right)=\max _{\lambda} L_{A}\left(p^{*}, \lambda\right)=L_{A}\left(p^{*}, \lambda^{*}\right)=C_{T}\left(p^{*}\right) \text {. }
$$

Os indivíduos primais $X_{u}=(p)^{u} ; u=1,2, \cdots, N_{u}$ devem evoluir para a solução ótima primal $X^{*}=p^{*}$. Na abordagem 
utilizada neste artigo, isto é feito em um processo evolutivo no grupo primal envolvendo uma busca irrestrita sobre um conjunto de funções Lagrangianas aumentadas $L_{A}\left(X_{u}, Y^{*}\right)$, (uma para cada indivíduo primal $u=1, \cdots, N_{u}$ ) calculadas para um valor específico de variável dual $Y^{*}$, conforme sugerido em (32). Como $Y^{*}$ não é conhecido pelos indivíduos do grupo primal, cada indivíduo deste grupo escolhe, em cada geração, o pior caso, ou seja, o indivíduo dual $Y^{*}=Y_{v}$ tal que $\max _{v} L_{A}\left(X_{u}, Y_{v}\right) \quad$ (estratégia de segurança), conforme já descrito. Supondo que cada indivíduo primal adota esta estratégia, tem-se um conjunto de $N_{u}$ funções Lagrangianas aumentadas, as quais poderiam ser utilizadas no cálculo do gap de dualidade. Neste artigo, propõe-se a definição de um valor médio para os valores das funções Lagrangianas aumentadas $L_{A}\left(X_{u}, Y^{*}\right)$ obtidas a partir dos jogos matriciais de todos os indivíduos, aqui denominada de Lagrangiana aumentada agrupada do grupo primal $\left(L_{A}^{P}\right)$, e definida conforme (33):

$$
L_{A}^{P}=\frac{\sum_{u=1}^{N_{u}} \max _{v} L_{A}\left(X_{u}, Y_{v}\right)}{N_{u}}
$$

No problema dual, um raciocínio análogo é adotado para a definição da função Lagrangiana aumentada agrupada para o grupo dual $\left(L_{A}^{D}\right)$, conforme mostrado em (34):

$$
L_{A}^{D}=\frac{\sum_{v=1}^{N_{v}} \min _{u} L_{A}\left((p)^{u}, Y_{v}\right)}{N_{v}}
$$

A equação (32) sugere que quando o valor de $L_{A}^{P}$ tende ao valor de $L_{A}^{D}$, tem-se uma solução primal-dual ótima para o problema de DE. Portanto, define-se aqui o gap de dualidade agrupado gap $_{g}$, conforme descrito em (35):

$$
\operatorname{gap}_{g}=L_{A}^{P}-L_{A}^{D}
$$

O critério de parada adotado neste trabalho para o procedimento co-evolutivo assume que gap $_{g}$ deve ser menor que uma determinada precisão $\varepsilon$ previamente definida. A experiência numérica obtida através deste trabalho mostra que quando gap $_{g} \rightarrow 0$ os indivíduos do grupo primal se adensam em um único ponto de solução primal, e os indivíduos duais também se adensam em torno de um único indivíduo dual. Assim, o valor de gap $_{g}$ tende ao valor de gap de dualidade tradicional. Apesar das evidências numéricas, estes resultados empíricos não são demonstrados matematicamente neste artigo.

\section{E. Mecanismo de Substituição}

O mecanismo de substituição consiste na substituição de um indivíduo aleatório do grupo pelo melhor indivíduo da última geração, de modo que o melhor indivíduo é sempre preservado em gerações futuras. Este mecanismo, que não está destacado na Fig. 1, também é denominado de elitismo na literatura, e é utilizado na abordagem aqui proposta para os grupos de indivíduos primal e dual. A seguir são descritos estudos de simulação envolvendo a metodologia de solução proposta.

\section{Resultados de Simulação}

As soluções calculadas pelo AGCE aqui proposto para o problema de DE, são comparadas com aquelas obtidas por outros métodos de otimização. Todos os métodos foram testados no ambiente desta pesquisa especificamente para comparações com o AGCE.

Os dados do sistema teste utilizado são descritos na subseção $A$. Na subseção $B$, a solução fornecida pelo AGCE é comparada às soluções obtidas pelos seguintes métodos: o método de pontos interiores (MPI) descrito em [6] e o algoritmo genético (AG) convencional (que trabalha apenas no subespaço primal do problema) com codificação em real, descrito em [2]. Na subseção $C$, são incluídos os chamados pontos de carregamento de válvula no problema de DE. Para este caso, a solução do AGCE é comparada apenas com a solução obtida pelo AG convencional descrito em [2], já que o MPI não é matematicamente aplicável, em função da não diferenciabilidade na função objetivo do DE. Na subseção $D$, é analisado o desempenho computacional do AGCE em relação ao AG convencional.

\section{A. Dados do Sistema Teste}

Para as simulações descritas a seguir, utilizou-se uma versão adaptada do sistema teste do IEEE 30 barras. Os parâmetros das unidades termelétricas conectadas às barras 1 , $2,5,8,11$ e 13 são mostrados na Tabela I, bem como os valores mínimos e máximos de geração de cada unidade $t$. Nota-se que as unidades conectadas às barras 8,11 e 13 são mais baratas do que as demais e não apresentam pontos de carregamento de válvula. A demanda total do sistema é de $D=283.4$.

TABELA I. PARÂMETROS DAS UNIDADES DE GERAÇÃO DO SISTEMA

\begin{tabular}{|c|c|c|c|c|c|c|c|}
\hline \hline$t$ & $\begin{array}{c}p_{t}^{\min } \\
{[M W]}\end{array}$ & $\begin{array}{c}p_{t}^{\max } \\
{[M W]}\end{array}$ & $\begin{array}{c}a_{t} \\
{\left[\$ / M W^{2}\right]}\end{array}$ & $\begin{array}{c}b_{t} \\
{[\$ / M W]}\end{array}$ & $\begin{array}{c}c_{t} \\
{[\$]}\end{array}$ & $e_{t}$ & $f_{t}$ \\
\hline \hline 1 & 0 & 160 & 0.040 & 6 & 600 & 50 & $0.03 \pi$ \\
\hline 2 & 0 & 60 & 0.040 & 5 & 500 & 25 & $0.08 \pi$ \\
\hline 5 & 0 & 80 & 0.025 & 4 & 400 & 20 & $0.04 \pi$ \\
\hline 8 & 0 & 50 & 0.028 & 0,34 & 0 & - & - \\
\hline 11 & 0 & 80 & 0.014 & 0,34 & 0 & - & - \\
\hline 13 & 0 & 60 & 0.028 & 0,28 & 0 & - & - \\
\hline
\end{tabular}

\section{B. Caso 1 - Formulação Quadrática para o DE}

Neste caso, a função não convexa dada em (23) é aproximada pela função quadrática, assumindo-se $e_{t}=f_{t}=0 ; t=1, \cdots, n t . \quad \mathrm{O}$ problema de $\mathrm{DE}$ quadrático resultante é resolvido pelo MPI, pelo AG convencional, e pelo AGCE aqui proposto.

A solução primal dual obtida pelo MPI é mostrada na Tabela II, que mostra o despacho das unidades $p_{t} ; t=\{1,2,5,8,11,13\} \quad$ em $\mathrm{MW}$, o valor de custo ótimo $C_{T}(p)$ em [\$] (unidade monetária padrão), o valor ótimo da 
função Lagrangiana aumentada $L_{A}$ em [\$], e o preço spot $p r$ (variável dual associada á restrição (21)) dado em $\$ / M W$. A precisão dos dados nesta e nas tabelas seguintes foi exagerada com o intuito de se verificar a precisão das soluções encontradas pelo AGCE. Como este problema de ED é quadrático, a solução ótima encontrada pelo MPI é o ótimo global do problema sendo, portanto, utilizada como a solução de referência para comparações com os outros métodos.

TABela II. Despacho de Geração, Custos e PREÇO CAlCULAdo PElo MPI PARA O CASO 1.

\begin{tabular}{|c|c|c|c|c|c|c|c|c|}
\hline \hline$p_{1}$ & $p_{2}$ & $p_{5}$ & $p_{8}$ & $p_{11}$ & $p_{13}$ & $C_{T}(p)$ & $L_{A}$ & $p r$ \\
\hline 11.3611 & 23.8611 & 58.1777 & 50.0000 & 80.0000 & 60.0000 & 2119.9269 & 2119.9269 & 6.90888 \\
\hline
\end{tabular}

O despacho de geração calculado pelo AG é mostrado na Tabela III para vários valores de tamanhos de população adotados. Os elementos da tabela são os mesmos já descritos na Tabela II. Observa-se que os valores de despacho obtidos pelo AG possuem precisão muito boa se comparados à solução de referência dada pelo MPI na Tabela II. Entretanto, é importante destacar que o AG não é capaz de resolver o subproblema dual já que seu processo evolutivo trabalha sobre as variáveis primais somente.

A solução fornecida pelo AGCE foi calculada utilizando-se uma população fixa de 20 indivíduos tanto para o grupo primal quanto para o grupo dual. Adotou-se uma precisão $\varepsilon=10^{-12}$ para o gap de dualidade agrupado gap $_{g}$ descrito em (35). Como o problema de DE é quadrático, para este caso adotou-se $\rho=0$ para o termo de penalidade quadrática na função Lagrangiana aumentada (24). Como o AGCE é um procedimento heurístico que envolve aleatoriedade, este algoritmo foi simulado 6 vezes.

TABELA III. DESPACHO DE GERAÇÃo E CUSTOS CALCULADO PELO AG PARA O

\begin{tabular}{|c|c|c|c|c|c|c|c|}
\hline $\begin{array}{c}\text { Tam. } \\
\text { pop. }\end{array}$ & $p_{1}$ & $p_{2}$ & $p_{5}$ & $p_{8}$ & $p_{11}$ & $p_{13}$ & $C_{T}(p)$ \\
\hline \hline 20 & 11.3611 & 23.8611 & 58.1778 & 50.0000 & 80.0000 & 60.0000 & 2119.9269 \\
\hline 40 & 11.3611 & 23.8611 & 58.1778 & 50.0000 & 80.0000 & 60.0000 & 2119.9269 \\
\hline 60 & 11.3611 & 23.8611 & 58.1778 & 50.0000 & 80.0000 & 60.0000 & 2119.9269 \\
\hline 80 & 11.3611 & 23.8611 & 58.1778 & 50.0000 & 80.0000 & 60.0000 & 2119.9269 \\
\hline 100 & 11.3611 & 23.8611 & 58.1778 & 50.0000 & 80.0000 & 60.0000 & 2119.9269 \\
\hline
\end{tabular}

Os resultados envolvendo as variáveis primais calculadas pelo AGCE são sintetizados na Tabela IV.

TABELA IV. VARIÁVEIS PRIMAIS CALCULADAS PELO AGCE PARA O CASO 1. \begin{tabular}{|c|c|c|c|c|c|c|c|c|}
\hline \hline Sim. & $p_{1}$ & $p_{2}$ & $p_{5}$ & $p_{8}$ & $p_{11}$ & $p_{13}$ & $C_{T}(p)$ & $L_{A}^{P}$ \\
\hline \hline 1 & 11.3611 & 23.8610 & 58.1778 & 50.0000 & 80.0000 & 60.0000 & 2119.9270 & 2119.9269 \\
\hline 2 & 11.3611 & 23.8610 & 58.1778 & 50.0000 & 80.0000 & 60.0000 & 2119.9270 & 2119.9269 \\
\hline 3 & 11.3611 & 23.8610 & 58.1778 & 50.0000 & 80.0000 & 60.0000 & 2119.9269 & 2119.9269 \\
\hline 4 & 11.3611 & 23.8610 & 58.1779 & 50.0000 & 80.0000 & 60.0000 & 2119.9266 & 2119.9269 \\
\hline 5 & 11.3611 & 23.8610 & 58.1778 & 50.0000 & 80.0000 & 60.0000 & 2119.9270 & 2119.9269 \\
\hline 6 & 11.3611 & 23.8611 & 58.1778 & 50.0000 & 80.0000 & 60.0000 & 2119.9269 & 2119.9269 \\
\hline
\end{tabular}

Enfatiza-se que $L_{A}^{P}$ corresponde à função Lagrangiana aumentada para o grupo primal, conforme descrito em (33). Os elementos restantes da Tabela são os mesmos dados na Tabela III. Analisando-se a Tabela IV verifica-se a boa precisão do despacho calculado quando comparado àquele dado pelo MPI na Tabela II. Também se observa que praticamente o mesmo resultado foi obtido em todas as 6 simulações, o que mostra a robustez do AGCE neste caso.

Os resultados relacionados às variáveis duais calculadas pelo AGCE nas 6 simulações são sintetizados na Tabela V, que mostra o preço spot $p r$ e a função Lagrangiana aumentada agrupada para o grupo dual $L_{A}^{D}$ para cada procedimento de simulação.

\begin{tabular}{|c|c|c|}
\hline \multicolumn{2}{|c|}{ TABELA V. VARIÁVEIS DUAIS CALCULADAS PELO AGCE PARA O CASO 1.} \\
\hline \hline Sim. & $p r$ & $L_{A}^{D}$ \\
\hline \hline 1 & 6.908887 & 2119.9269 \\
\hline 2 & 6.908890 & 2119.9269 \\
\hline 3 & 6.908882 & 2119.9269 \\
\hline 4 & 6.908896 & 2119.9269 \\
\hline 5 & 6.908890 & 2119.9269 \\
\hline 6 & 6.908890 & 2119.9269 \\
\hline
\end{tabular}

Comparando-se os resultados da Tabela $\mathrm{V}$ com aqueles mostrados na Tabela IV, observa-se que as funções Lagrangianas aumentadas agrupadas calculadas para os grupos primais e duais, dadas respectivamente por $L_{A}^{P}$ e $L_{A}^{D}$, convergem de forma consistente para o mesmo valor, o qual corresponde ao valor dos custos de geração $C_{T}(p)$ em todos os 6 procedimentos de simulação. Este resultado confirma a existência de um ponto de sela para a função Lagrangiana aumentada que resolve o problema de DE. A evolução do gap de dualidade agrupado gap $_{g}$ definido em (35) é mostrada na Figura 3.

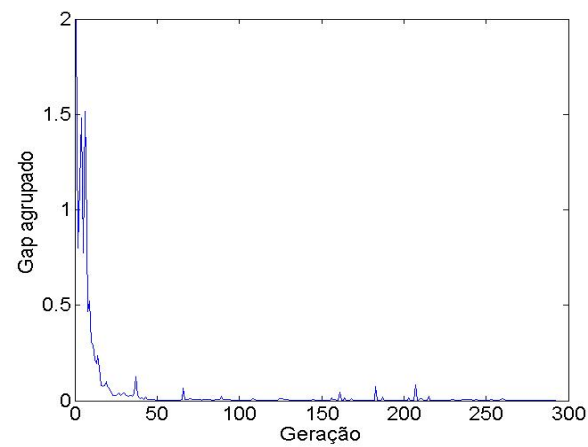

Figura 3. Evolução do Gap de dualidade agrupado em cada geração.

O valor final do gap $_{g}$ é menor do que $\varepsilon=10^{-12}$, e este tende ao valor do gap de dualidade tradicional definido na teoria de otimização clássica. Nota-se ainda que o preço spot pr também foi obtido em todas as simulações com boa precisão, se comparado ao valor de referência calculado pelo MPI na Tabela II.

É importante enfatizar que, diferentemente das abordagens de AG convencionais, na abordagem do AGCE as variáveis duais são efetivamente calculadas no processo evolutivo. Outra vantagem importante é que a solução primal-dual foi calculada pelo AGCE com uma precisão pré-especificada e que o critério de parada envolve a distância pré-especificada a um ponto de sela que resolve o problema de DE. 


\section{Caso 2 - Formulação Não Convexa par o DE}

Neste estudo de caso, os efeitos de carregamento de pontos de válvula são levados em consideração pela introdução dos valores dos parâmetros $e_{t}, f_{t} ; t=\{1,2,5\}$ fornecidos na Tabela I. Neste caso, o problema de DE torna-se não convexo e não diferenciável e com vários pontos de mínimo e máximo locais, conforme mostrado na Figura 2. Em função da não diferenciabilidade, as condições de Karush-Kuhn-Tucker (KKT) não se aplicam neste caso. Além disso, os métodos convencionais de otimização, os quais são geralmente baseados em variações sobre a direção do gradiente não são aplicáveis. Portanto o MPI não pode ser utilizado para resolver o problema, o qual é resolvido nesta seção somente pelo AG convencional e pelo AGCE.

Neste caso 2, a função Lagrangiana aumentada possui vários pontos de sela, devido à presença de vários pontos de mínimo e máximo local. Portanto, o AGCE foi simulado muitas vezes, de modo a mapear os possíveis pontos de sela, os quais são candidatos à solução global do problema. As 6 melhores soluções obtidas pelo AGCE são mostradas na Tabela VI, que é idêntica em estrutura à Tabela IV.

TABELA VI. VARIÁVEIS PRIMAIS CALCULADAS PELO AGCE PARA O CASO 2.
\begin{tabular}{|c|c|c|c|c|c|c|c|c|}
\hline \hline $\begin{array}{c}\text { Sim } \\
.\end{array}$ & $p_{1}$ & $p_{2}$ & $p_{5}$ & $p_{8}$ & $p_{11}$ & $p_{13}$ & $C_{T}(p)$ & $L_{A}^{P}$ \\
\hline \hline 1 & $\begin{array}{c}0.000 \\
0\end{array}$ & 25.0000 & 68.4000 & 50.0000 & 80.0000 & 60.0000 & $\begin{array}{c}2191.19958 \\
3\end{array}$ & $\begin{array}{c}2191.19956 \\
5\end{array}$ \\
\hline 2 & $\begin{array}{c}0.000 \\
0\end{array}$ & 25.0000 & 68.4000 & 50.0000 & 80.0000 & 60.0000 & $\begin{array}{c}2191.19958 \\
3\end{array}$ & $\begin{array}{c}2191.19956 \\
5\end{array}$ \\
\hline 3 & $\begin{array}{c}0.000 \\
0\end{array}$ & 25.0000 & 68.4000 & 50.0000 & 80.0000 & 60.0000 & $\begin{array}{c}2191.19958 \\
3\end{array}$ & $\begin{array}{c}2191.19956 \\
5\end{array}$ \\
\hline 4 & $\begin{array}{c}0.000 \\
0\end{array}$ & 25.0000 & 68.4000 & 50.0000 & 80.0000 & 60.0000 & $\begin{array}{c}2191.19958 \\
3\end{array}$ & $\begin{array}{c}2191.19956 \\
5\end{array}$ \\
\hline 5 & $\begin{array}{c}0.000 \\
0\end{array}$ & 25.0000 & 68.4000 & 50.0000 & 80.0000 & 60.0000 & $\begin{array}{c}2191.19958 \\
3\end{array}$ & $\begin{array}{c}2191.19956 \\
5\end{array}$ \\
\hline 6 & $\begin{array}{c}0.000 \\
0\end{array}$ & 25.0000 & 68.4000 & 50.0000 & 80.0000 & 60.0000 & $\begin{array}{c}2191.19958 \\
3\end{array}$ & $\begin{array}{c}2191.19956 \\
5\end{array}$ \\
\hline
\end{tabular}

Observa-se que os valores da função Lagrangiana aumentada agrupada para indivíduos do grupo primal $L_{A}^{P}$ tendem aos custos totais $C_{T}(p)$ com boa precisão em todos os 6 procedimentos de simulação. Observa-se que os custos totais são mais altos que aqueles obtidos no caso 1 , o que já era esperado, já que o efeito de carregamento de pontos válvula tende a aumentar os custos, conforme pode ser observado na Figura 2.

Devido às não linearidades nos custos, alguns ajustes nos parâmetros do AGCE foram feitos de modo a obter a solução mostrada na Tabela VI, tais como: a precisão do gap de dualidade agrupado foi reduzida para $\varepsilon=10^{-16}$; adotou-se um termo de penalidade adaptativa $\rho$, o qual foi aumentado de forma gradual no processo iterativo, utilizando-se a expressão $\rho^{k+1}=1.0005 \rho^{k}$, em que $k$ é o número da iteração (geração) atual. Esta estratégia melhorou a precisão das soluções obtidas, além de facilitar a identificação dos pontos de sela associados ao ótimo global do problema de DE. Note que para esta solução manteve-se o número de 20 indivíduos para as populações primal e dual.

OS RESULTADO ASSOCIADOS ÀS VARIÁVEIS DUAIS CALCULADAS PELO AGCE EM TODAS AS 6 SIMULAÇÕES PARA O CASO 2 ESTÃO SINTETIZADOS NA

Tabela VII.
TABELA VII. VARIÁVEIS DUAIS CALCULADAS PELO AGCE PARA O CASO 2.

\begin{tabular}{|c|c|c|}
\hline \hline Sim. & $p r$ & $L_{A}^{D}$ \\
\hline \hline 1 & 10.76745705 & 2191.199565 \\
\hline 2 & 10.76745705 & 2191.199565 \\
\hline 3 & 10.76745705 & 2191.199565 \\
\hline 4 & 10.76745705 & 2191.199565 \\
\hline 5 & 10.76745705 & 2191.199565 \\
\hline 6 & 10.76745705 & 2191.199565 \\
\hline
\end{tabular}

Pela comparaÇão entre as Tabelas VI e VII, observa-Se Que $L_{A}^{P}$ E

$L_{A}^{D}$ CONVERGEM DE FORMA CONSISTENTE PARA O MESMO VALOR, O QUAL É

IDÊNTICO AO VALOR DOS CUSTOS TOTAIS $C_{T}(p)$ EM TODOS OS 6

PROCEDIMENTOS DE SOLUÇÃO AVALIADOS. A PRECISÃO OBTIDA NESTE CASO É MUITO BOA DEVIDO Ả REDUÇÃO NA PRECISÃO DO GAP DE DUALIDADE AGRUPADO. MAIS UMA VEZ, ESTE RESULTADO CONFIRMA A EXISTÊNCIA DE UM PONTO DE SELA PARA A FUNÇÃO LAGRANGIANA AUMENTADA, O QUAL RESOLVE O PROBLEMA DE DE. NOTE QUE O PONTO DE SELA É IDENTIFICADO MESMO EM UMA SITUAÇ̃̃O EM QUE A FUNÇ̃̃O OBJETIVO NÃO É

DIFERENCIÁVEL. PORTANTO, O AGCE FOI CAPAZ DE IDENTIFICAR PONTOS DE SOLUÇÃO ÓTIMOS MESMO PARA UMA SITUAÇÃO EM QUE AS CONDIÇÕES DE KKT NÃO SÃO APLICÁVEIS. VALE DESTACAR AINDA QUE O PREÇO SPOT FOI CONSISTENTEMENTE OBTIDO EM TODOS OS 6 PROCESSOS DE SIMULAÇÃO MOSTRADOS NA

Tabela VII. O preço final obtido é maior que aquele obtido para o caso 1 , o que é um resultado esperado, já que os custos gerais aumentaram no caso 2 , causando assim um aumento no preço da energia.

O problema de DE também foi resolvido pela AG convencional. O despacho calculado pelo AG é resumido na Tabela VIII para vários valores de tamanho de população adotado. Devido às não-linearidades, foi necessário aumentar o tamanho da população para que o AG tivesse uma melhor precisão, quando comparado à solução de referência fornecida pelo AGCE. Observa-se, por exemplo, que a solução obtida pelo AG com um tamanho de população de 40 indivíduos não foi muito precisa se comparada à solução do AGCE. Para valores maiores de tamanho populacional, a solução do problema primal do $\mathrm{AG}$ tende à solução calculada pelo AGCE. A solução dual, entretanto, não pode ser calculada pela abordagem de AG convencional.

TABEla VIII. DESPACHO DE GERAÇÃo E CUSTOS CALCULAdO PELO AG PARA

\begin{tabular}{|c|c|c|c|c|c|c|c|}
\hline \hline Tam.Pop. & $p_{1}$ & $p_{2}$ & $p_{5}$ & $p_{8}$ & $p_{11}$ & $p_{13}$ & $C_{T}(p)$ \\
\hline \hline 40 & 0.9000 & 12.5000 & 80.0000 & 50.0000 & 80.0000 & 60.0000 & 2206.0595 \\
\hline 60 & 0.0000 & 24.9999 & 68.4000 & 50.0000 & 80.0000 & 60.0000 & 2191.1997 \\
\hline 80 & 0.0000 & 25.0000 & 68.4000 & 50.0000 & 80.0000 & 60.0000 & 2191.1996 \\
\hline 100 & 0.0000 & 25.0000 & 68.4000 & 50.0000 & 80.0000 & 60.0000 & 2191.1995 \\
\hline
\end{tabular}

\section{Desempenho Computacional}

Nesta seção é feita uma análise conceitual e numérica do desempenho computacional do AGCE quando comparado ao AG convencional.

Pelo fato de o método envolver dois grupos de populações, pode-se, a princípio, imaginar que este método possua tempos computacionais muito mais elevados que aqueles obtidos para os métodos tradicionais (os quais evoluem apenas uma população de indivíduos). Entretanto, em [18] mostra-se que isso de fato não ocorre. Para isso, sejam os grupos primais e duais, dados em (16) e (17). A abordagem co-evolutiva necessita calcular $L_{A}\left(u_{i}, v_{j}\right)$ para $\forall i=1, \cdots, N_{u}$ e para 
$\forall j=1, \cdots, N_{v}$. Assim o número de cálculos de $L_{A}$ é $N_{u} \times N_{v}$. Entretanto, o cálculo de $f(x), g(x)$ e $h(x)$ é feito apenas $N_{u}$ vezes, uma para cada $u_{i}$. Dado que $f(x), g(x)$ e $h(x)$ são calculadas para um dado $u_{i}$, o valor de $L_{A}\left(u_{i}, v_{j}\right)$ para cada $Y_{j}$ requer apenas o cálculo de $\mu^{T} g(x)+\lambda^{T} h(x)$ e dos demais termos de penalidade. É importante notar que o cálculo do custo e das restrições consome a maior parte do tempo computacional de problemas práticos. Assim, o esforço computacional do método co-evolutivo aqui descrito é maior, porém comparável àquele dos métodos baseados na evolução de apenas um único grupo.

Os resultados numéricos envolvendo a comparação do desempenho computacional do AGCE e do AG convencional são sintetizados na Tabela IX. É importante enfatizar que o AGCE foi implementado em $\mathrm{C}++$ enquanto que o $\mathrm{AG}$ convencional e o MPI foram implementados em MATLAB, com objetivos iniciais de validação das soluções ótimas primal e dual.

TABELA IX

SÍNTESE DA PERFORMANCE COMPUTACIONAL PARA O AGCE E AG CONVENCIONAL.

\begin{tabular}{|c|c|c|c|c|c|c|c|}
\hline \multirow[t]{2}{*}{ Sim. } & \multicolumn{2}{|c|}{$\begin{array}{l}\text { Número de } \\
\text { Gerações }\end{array}$} & \multicolumn{2}{|c|}{$\begin{array}{l}\text { Tempo total de } \\
\text { CPU (s) }\end{array}$} & \multicolumn{2}{|c|}{$\begin{array}{c}\text { Tempo de CPU para uma } \\
\text { geração (s) }\end{array}$} & \multirow{2}{*}{\begin{tabular}{|c}
$\begin{array}{c}\text { Valor de penal. } \\
\text { final }\end{array}$ \\
AGCE \\
\end{tabular}} \\
\hline & $\mathrm{AG}$ & AGCE & $\mathrm{AG}$ & AGCE & $\mathrm{AG}$ & AGCE & \\
\hline 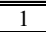 & 1000 & 8609 & 7.41 & 13.00 & 0.007407 & 0.001510 & 0.153793 \\
\hline 2 & 1000 & 9897 & 7.39 & 15.00 & 0.007390 & 0.001516 & 0.164023 \\
\hline 3 & 1000 & 13976 & 7.52 & 21.00 & 0.007515 & 0.001503 & 0.201130 \\
\hline 4 & 1000 & 3628 & 7.47 & 6.00 & 0.007468 & 0.001654 & 0.119889 \\
\hline 5 & 1000 & 5683 & 7.42 & 9.00 & 0.007422 & 0.001584 & 0.132862 \\
\hline 6 & 1000 & 7915 & 7.48 & 12.00 & 0.007484 & 0.001516 & 0.148548 \\
\hline & 1000 & 8284.6 & 7.45 & 12.67 & 0.007448 & 0.001547 & 0.153374 \\
\hline
\end{tabular}

Os testes foram feitos em um processador Intel ${ }^{\circledR}$ Core $^{\mathrm{TM}} 2$ duo CPU E700 $2.53 \mathrm{GHz}$, em um sistema operacional Windows Vista operacional system, com $4 \mathrm{Mb}$ RAM. Utilizou-se a versão DEV C++ 4.9.9.2 para a implementação do AGCE e o MATLAB 7.10.0.499 (R2010a) de 32 bits para programar o AG convencional. Fixou-se o tamanho da população para o AGCE e AG em 20 indivíduos. Ambos os algoritmos foram simulados 6 vezes e os resultados mostrados na Tabela IX são referentes ao caso 2 (descrito na seção anterior). A Tabela IX mostra o número de gerações, o tempo total de CPU, e o tempo médio de CPU para processar uma única geração, e o valor de penalidade final utilizado para o AGCE em cada simulação. Valores médios são mostrados na parte de baixo da tabela.

Observa-se que para cada simulação mostrada na Tabela IX, o AGCE gasta um número de gerações diferente para reduzir o gap de dualidade agrupado até a precisão de $10^{-14}$ adotada em todas as simulações. $\mathrm{O}$ valor médio de gerações para atingir a convergência é de 8284 , ao contrário do AG convencional, que possui um número de gerações préespecificado (1000). Também se pode observar que para atingir a precisão estabelecida, o AGCE geralmente necessita um número de gerações maior do que o AG convencional. É importante destacar que o $\mathrm{AG}$ convencional não obteve soluções precisas nas 6 simulações feitas, devido ao pequeno número de indivíduos adotados na população.
Conforme comentado anteriormente, o esforço computacional do AGCE deve ser um pouco maior, porém comparável ao esforço do AG convencional. Entretanto, mostra-se na Tabela IX que o tempo para calcular uma única geração é aproximadamente 5 vezes maior para o AG do que para o AGCE. Isso ocorre pelo fato de que o AGCE foi implementado em $\mathrm{C}++$, enquanto que o $\mathrm{AG}$ foi implementado em MATLAB, cujo desempenho computacional é sabidamente inferior ao da linguagem $\mathrm{C}++$. Repara-se, ainda na Tabela que os valores finais de penalidade utilizados para tornar a função objetivo convexa são pequenos em todas as simulações.

É importante destacar que o foco deste trabalho é mostrar que o AGCE é capaz de calcular uma solução primal dual exata. Investigações adicionais são necessárias de modo a melhorar o desempenho do AGCE, bem como para avaliar sua aplicabilidade à solução de problemas de despacho de geração de grande porte.

\section{CONCLUSÕES}

Este trabalho propõe um algoritmo genético co-evolutivo (AGCE) baseado na função Lagrangiana aumentada para a solução do problema de despacho econômico (DE). O DE se torna não convexo e não diferenciável quando os efeitos de pontos de carregamento de válvula são considerados na função de produção de unidades de geração termelétricas. Nestes casos, algoritmos evolutivos clássicos têm sido tradicionalmente utilizados para resolver o problema primal de DE. Entretanto, estes algoritmos geralmente não são capazes de resolver o problema dual associado ao DE. Nos resultados obtidos com um sistema teste, o AGCE foi capaz de calcular os problemas primal e dual relacionados ao DE, mesmo para situações de não diferenciabilidade. Além disso, propõe-se neste trabalho um critério de parada para o AGCE que está baseado na otimalidade, e em uma precisão adotada para encontrar a solução ótima, o que não é possível em algoritmos evolutivos convencionais, cujo critério de parada é baseado em um número fixo de gerações. Nos resultados obtidos para formulações quadráticas do $\mathrm{DE}$, o AGCE obteve valores primais e duais idênticos àqueles obtidos pelo método de pontos interiores (MPI), utilizado como referência. Mesmo nos casos em que o DE foi formulado de forma mais precisa, inserindo-se a representação por pontos de válvula, resultando em um problema de DE não convexo e não diferenciável, o AGCE foi capaz de calcular a solução dos problemas primal e dual. Neste caso, a solução do problema primal foi comparada à solução obtida pelo $\mathrm{AG}$ convencional com boa precisão. Portanto, os resultados mostraram que o AGCE foi capaz de identificar soluções ótimas, por meio da busca de pontos de sela na função Lagrangiana aumentada, mesmo em situações em que as condições de KKT não podem ser aplicadas, em função da não diferenciabilidade. Investigações adicionais são necessárias de modo a avaliar e comparar de forma mais adequada o desempenho computacional do AGCE com os AGs convencionais, bem como para verificar a aplicabilidade da metodologia para a solução de problemas de DE para sistemas de grande porte. 


\section{AGRADECIMENTOS}

Os autores agradecem à FAPESP e ao CNPq pelo auxílio financeiro concedido através dos processos 2010/12479-0 e $308531 / 2009$.

\section{REFERÊNCIAS}

[1] Amjady, N.; Nasiri-Rad, H "Economic dispatch using an efficient realcoded genetic algorithm," IET Generation, Transmission \& Distribution, vol. 3 , no. 3, pp. $266-278,2009$.

[2] Barbosa, D. R.; Silva, A. L; Luciano, E. J. R.; Nepomuceno, L. "Uma Abordagem Baseada em Algoritmos Genéticos com Codificação em Real para a Solução de um FPO DC para Sistemas Hidrotérmicos," The 8th Latin-American Congress on Electricity, Generation and Transmission - CLAGTEE, 2009

[3] Bazaraa, M. S.; Sheraly, H. D.; Shetty, C. M. "Nonlinear ProgrammingTheory and Algorithms," $3^{\text {rd }}$ edition, John Wiley \& Sons, 2006.

[4] Basu, M. "Artificial bee colony optimization for multi-area economic dispatch," International Journal of Electrical Power \& Energy Systems, vol. 49, pp. 181-187, 2013.

[5] Ciornei, I.; Kyriakides, E. "A GA-API Solution for the Economic Dispatch of Generation in Power System Operation," IEEE transactions on power systems, vol. 27, no. 1, pp. 233-242, 2012.

[6] Costa, L. H.; Silva A. L., Vergílio, A. H. B.; Nepomuceno, L. "Um Modelo de Despacho Econômico Hidrotérmico para os Mercados de Energia Utilizando o Método de Pontos Interiores," The 8th LatinAmerican Congress on Electricity, Generation and Transmission CLAGTEE, 2009.

[7] Ghasemi, A. "A fuzzified multi-objective interactive honey bee mating optimization for environmental/economic power dispatch with valve point effect," International Journal of Electrical Power \& Energy Systems, vol. 49, pp. 308-321, 2013.

[8] Hemamalini, S.; Simon, S. P. "Dynamic economic dispatch using artificial immune system for units with valve-point effect," International Journal of Electrical Power \& Energy Systems, vol. 33, no. 4, pp. 868874., 2011.

[9] Hestenes, M. R. "Survey paper - Multiplier and Gradient Methods," Journal of Optimization Theory and Applications, vol. 4, no. 5, pp. 303$320,1969$.

[10] Lee, J.-C., Lin, W.-M.; Liao, G.-C. Tsao, T.-P. "Quantum genetic algorithm for dynamic economic dispatch with valve-point effects and including wind power system," International Journal of Electrical Power \& Energy Systems, vol. 33, no. 2, pp. 189-197, 2011.

[11] Meng, K; Wang, H. G.; Dong, Z. Y.; Wong, K. P. "Quantum-Inspired Particle Swarm Optimization for Valve-Point Economic Load Dispatch," IEEE Transactions on Power Systems, vol. 25, no. 1, pp. 215 $-222,2001$

[12] Oliveira, A. R. L.; Soares, S.; Nepomuceno, L. “Optimal Active Power Dispatch Combining Network Flow and Interior Point Approaches,' IEEE Transactions on Power System, vol. 18, no. 4, pp. 1235-1240, 2003.

[13] Powell, M. J. D. "A Method for Nonlinear Constraints in Minimization Problems. In: Fletcher, R. Optimization”, New York, Academic Press, pp. 283-298, 1969.

[14] Reddy, A. S.; Vaisakh, K. "Shuffled differential evolution for economic dispatch with valve point loading effects," International Journal of Electrical Power \& Energy Systems, vol. 46, pp. 342-352, 2013.
[15] Rockafellar, R. T. "The Multiplier Method of Hestenes and Powell Applied to Convex Programming," Journal of Optimization Theory and Applications, vol. 12, no. 6, pp. 555-562, 1973.

[16] Sa-ngiamvibool, W.; Pothiya, S.; Ngamroo, I. "Multiple tabu search algorithm for economic dispatch problem considering valve-poin effects," International Journal of Electrical Power \& Energy Systems, vol. 33 , no. 4 , pp. 846-854, 2011.

[17] Silva, I. N.; Nepomuceno, L., Bastos, T. M. "An efficient Hopfield network to solve economic dispatch problems with transmission system representation," Electrical Power and Energy Systems, vol. 26, pp. 733738, 2004.

[18] Tahk, M. J.; Sun, B. C. "Coevolutionary Augmented Lagrangian Methods for Constrained Optimization". IEEE Transactions on Evolutionary Computation, vol. 4, no. 2, pp. 114-124, 2000.

[19] Venkatesh, P.; Gnanadass, R.; Padhy, N.P. "Comparison and application of evolutionary programming techniques to combined economic emission dispatch with line flow constraints," IEEE Transactions on Power Systems, vol. 18, no. 2. pp. 688 - 697, 2003.

[20] Wong, K. P.; Fung, C.C. "Simulated annealing based economic dispatch algorithm”, IEE proceedings-C, vol. 140, no. 6, pp. 509-515, 1993.

[21] Wong, K. P.; Wong, S. Y. W. "Combined genetic algorithm/simulated annealing/fuzzy set approach to short-term generation scheduling with take-or-pay fuel contracts," IEEE Trans. Power Syst., vol. 11, no. 11, pp. 128-136, 1996.

[22] Yang, H. T.; Yang, P. C.; Huang, C. L. "Evolutionary programming based economic dispatch for units with nonsmooth fuel cost functions," IEEE Trans. Power Syst., vol. 11, pp. 112-118, 1996.

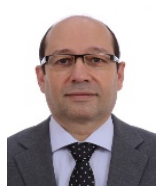

Leonardo Nepomuceno, é graduado em Engenharia Elétrica pela Universidade Federal de Uberlândia - UFU- MG, Brasil, em 1990, tendo obtido os títulos de mestre e doutor em Engenharia Elétrica pela Universidade Estadual de Campinas - UNICAMP, Campinas - SP, Brasil, respectivamente em 1993 e 1997. No período de 1997 a 2000, o professor atuou em projeto de pós-doutorado na UNICAMP. Atualmente, o professor desenvolve pesquisas na Universidade Estadual Paulista - UNESP Bauru - SP, nas áreas de planejamento e otimização de sistemas de energia e em mercados de energia.

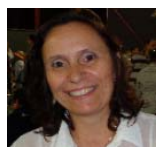

Edméa Cássia Baptista, é graduada em Matemática pela Universidade Estadual Paulista - UNESP - SP, em 1988 Universidade Estadual Paulista - UNESP - SP, em 1988,
tendo obtido os títulos de mestre e doutora em Engenharia Elétrica pela Universidade de São Paulo - USP, em 1993 e 2001, respectivamente. Atualmente é Professora Adjunta do Departamento de Matemática da UNESP, em Bauru, onde desenvolve pesquisas nas áreas de otimização, programação não linear, fluxo de potencia ótimo e planicidade.

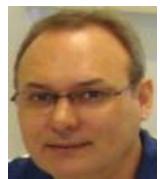

Antonio Roberto Balbo, é graduado em Matemática Universidade Estadual Paulista - UNESP - SP, em 1986, tendo obtido seu título de mestre em Ciência da Computação, em 1991 e seu título de doutor em Engenharia de Estruturas em 1998, ambos pela Universidade de São Paulo - USP Atualmente é Professor Adjunto do Departamento de Matemática da UNESP, em Bauru, onde desenvolve pesquisas nas áreas de métodos primal-dual previsor-corretor de pontos interiores/exteriores aplicados em sistemas de energia.

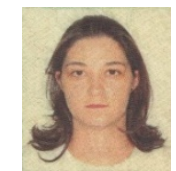

Edilaine Martins Soler, é graduada em Matemática pela Universidade de São Paulo - USP, em 2007, tendo obtido tendo obtido seu título de mestre em Ciência da Computação e Matemática Computacional, em 2008, e seu título de doutora em Engenharia Elétrica, em 2011, ambos pela Universidade de São Paulo - USP. Atualmente é Professora Assistente da Universidade Estadual Paulista - UNESP - SP, onde desenvolve pesquisas nas áreas de otimização, atuando principalmente nos seguintes temas: programação não linear, variáveis discretas e problema de fluxo de potência ótimo. 